\title{
NMR based metabolomics revealed Underlying Inflammatory Pathology in Reactive arthritis Synovial Joints
}

Durgesh Dubey ${ }^{1,2, \$,}{ }^{*}$, Sandeep Kumar $2, \$$, Atul Rawat ${ }^{1}$, Anupam Guleria ${ }^{1}$, Reena Kumari ${ }^{4}$, Sakir Ahmed ${ }^{2,5}$,

Rajeev Singh ${ }^{3}$, Ramnath Misra2, $5^{*}$ Dinesh Kumar ${ }^{1^{*}}$

${ }^{1}$ Centre of Biomedical Research, Lucknow-226014, India

2Department of Clinical Immunology \& Rheumatology, SGPGIMS, Lucknow-226014, India

${ }^{3}$ Regional Medical Research Center, Gorakhpur-273013, India

4Department of Biochemistry, KGMU, Lucknow-226003, India

5Department of Clinical Immunology and Rheumatology, KIMS, Bhubaneswar-751024, India

\$Authors contributed equally

\section{*Authors for Correspondence}

\author{
Dr. Durgesh Dubey \\ Department of Clinical Immunology \& Rheumatology, \\ SGPGIMS, Lucknow-226014 \\ Uttar Pradesh, India \\ Mobile: +919450784933 \\ ORCID: 0000-0002-7360-4994 \\ Email: durgeshcbmr@gmail.com
}

\author{
Dr. Ramnath Misra \\ (Professor and Head) \\ Department of Clinical Immunology and Rheumatology \\ Kalinga Institute of Medical Sciences, \\ Bhubaneswar, INDIA-751024 \\ ORCID: 0000-0001-7921-4199 \\ Mobile: +91-9415006688 \\ Email: rnmisra2000@gmail.com
}

\author{
Dr. Dinesh Kumar \\ (Associate Professor) \\ Centre of Biomedical Research (CBMR), \\ SGPGIMS Campus, Raibareli Road, \\ Lucknow-226014 Uttar Pradesh, India \\ Mobile: +91-8953261506 \\ ORCID: 0000-0001-8079-6739 \\ Email: dineshcbmr@gmail.com; dinesh@cbmr.res.in
}

Keywords: NMR, Metabolomics, Synovial Fluid, Rheumatic Diseases (RD), Rheumatoid Arthritis, Reactive Arthritis, Biomarkers, Undifferentiated Spondyloarthropathy 


\section{Table of Contents}

Supplementary Table S1: Summary table for the metabolite set enrichment analysis (MSEA) are ranked by Holm p-value. The Holm p-value is the p-value adjusted by the Holm-Bonferroni test, which is a tool used for largescale data analysis to counteract the issue of multiple comparisons. At left side, the yellow to red colors summarizes metabolic pathways that were lowest to strongest affected respectively in the ReA compared to OA patients.....S3

Supplementary Table S2: Summary table for the metabolite set enrichment analysis (MSEA) are ranked by Holm p-value. The Holm p-value is the p-value adjusted by the Holm-Bonferroni test, which is a tool used for largescale data analysis to counteract the issue of multiple comparisons. At left side, the yellow to red colors summarizes metabolic pathways that were lowest to strongest affected respectively in the ReA compared to RA patients....S5

Supplementary Table S3: Pearson correlation table $(n=38)$ of metabolites present in SF and serum....S6

Supplementary Table S4: Significnat metabolites of ReA compare to non-ReA groupswith p-value, false discovery rate (FDR).....S8

Supplementary Figure S1: Representative $800 \mathrm{MHz}{ }^{1} \mathrm{H}$ NMR spectra $\delta(0.75-4.65)(\mathrm{A})$ and $\delta(5.2-8.6)$ (B) of synovial fluid obtained from the OA, RA and ReA groups. The region of $\delta(5.2-8.5)$ is magnified 8 times compared with the corresponding region of $\delta(0.5-4.7)$ for the purpose of clarity. Abbreviations and notions used are: 3-HB: 3Hydroxybutyrate; NAG: N-acetylated glycoprotein; LDL: Low Density Lipoprotein; VLDL: Very Low Density Lipoprotein; PUFA: Polyunsaturated Fatty Acid $-\mathrm{C} \underline{\mathrm{H}}=\mathrm{C} \underline{\mathrm{H}}-; \quad \mathrm{L} 1:-\mathrm{C}_{2}-\mathrm{CH}_{2}-\mathrm{C}=\mathrm{O}-; \quad \mathrm{L} 2:-\mathrm{C}_{2}{ }^{-} \mathrm{C}=\mathrm{O}-; \quad \mathrm{L} 3:=\mathrm{CH}-\mathrm{C}_{2}{ }^{-}$ $\mathrm{CH}=\mathrm{C}-\ldots . . \mathrm{S} 9$

Supplementary Figure S2: The statistical validation plots (permutation number $=100$ ) for OPLS-DA model based discriminatory analysis (A) between $\operatorname{ReA}$ and $R A\left(R^{2} X(\right.$ cum $)=0.739, R^{2} Y($ cum $)=0.671$, and $Q^{2}($ cum $\left.)=0.514\right)$. (B) Between $\operatorname{ReA}$ and $O A\left(R^{2} X(\right.$ cum $)=0.723, R^{2} Y($ cum $)=0.754$, and $Q^{2}($ cum $\left.)=0.0 .604\right)$...S10

Supplementary Figure S3: Significantly altered metabolic pathways in ReA patient compare to OA and RA. All matched pathways are shown according to $p$ values from the pathway enrichment analysis ( $y$ axis) and pathway impact values from the pathway topology analysis ( $x$ axis). Colour gradient and circle size indicate the significance of the pathway ranked by $p$ value (yellow: higher $p$ values and red: lower $p$ values) and pathway impact values (the larger the circle the higher the impact score). Only the significantly affected pathways with low $p$ value and high pathway impact score are showed...S10.

Supplementary Figure S4: Metabolic pathway analyses related to the metabolites that significantly differ in ReA compared to nor-ReA group, utilizing the MetaboAnalyst functional interpretation tools. Panel A, graphic 
summary of metabolite set enrichment analysis for ReA vs OA groups. Panel B, graphic summary of metabolite set enrichment analysis for ReA vs RA groups. The horizontal bars summarize the main metabolite sets identified in this analysis; the bars are coloured based on their p-values and the length is based on the -fold enrichment...S11

Supplementary Figure S5: Potential biomarkers of (A) ReA vs OA and (B) ReA vs RA predicted by classical univariate ROC curve analysis. The left-side plot of the individual metabolite figure shows a 95\% confidence interval (shadowed) ROC (receiver operating characteristics) curve and the solid red dot shows the optimum cutoff with the corresponding sensitivity and specificity values. The right-side box-and-whisker plot shows the distribution of abundance values of individual metabolite in ReA compare to non-ReA group with the optimal cutoff as a horizontal dotted red line...S12

Supplementary Figure S6: The percentage selected frequency of metabolites based on ROC curves, with the variable importance in projection (VIP) plot from ReA vs RA (A) and ReA vs RA (C) indicating the most discriminating metabolite in descending order of importance; prediction of ReA patients from (B) RA and (D) OA groups using Monte-Carlo cross validation (MCCV) analysis. The class membership of the left-out sample was predicted using an a priori cut-off value of 0.5 (dashed line)....S13 


\section{Supplementary}

Table S1: Summary table for the metabolite set enrichment analysis (MSEA) are ranked by Holm p-value. The Holm p-value is the p-value adjusted by the Holm-Bonferroni test, which is a tool used for largescale data analysis to counteract the issue of multiple comparisons. At left side, the yellow to red colors summarizes metabolic pathways that were lowest to strongest affected respectively in the ReA compared to OA patients.

\begin{tabular}{|c|c|c|c|c|c|c|}
\hline Metabolite Set & $\begin{array}{c}\text { Tota } \\
1\end{array}$ & Hits & Expect & $P$ value & Holm P & FDR \\
\hline Glucose-Alanine Cycle & 13 & 3 & 0.114 & $1.29 \mathrm{E}-4$ & 0.0126 & 0.0126 \\
\hline Alanine Metabolism & 17 & 2 & 0.149 & 0.00873 & 0.846 & 0.428 \\
\hline Glutathione Metabolism & 21 & 2 & 0.185 & 0.0132 & 1.0 & 0.432 \\
\hline Urea Cycle & 29 & 2 & 0.255 & 0.0247 & 1.0 & 0.492 \\
\hline Amino Sugar Metabolism & 33 & 2 & 0.29 & 0.0315 & 1.0 & 0.492 \\
\hline Aspartate Metabolism & 35 & 2 & 0.308 & 0.0352 & 1.0 & 0.492 \\
\hline Fatty Acid Biosynthesis & 35 & 2 & 0.308 & 0.0352 & 1.0 & 0.492 \\
\hline Galactose Metabolism & 38 & 2 & 0.334 & 0.041 & 1.0 & 0.502 \\
\hline Glutamate Metabolism & 49 & 2 & 0.431 & 0.0651 & 1.0 & 0.609 \\
\hline Lactose Degradation & 9 & 1 & 0.0791 & 0.0767 & 1.0 & 0.609 \\
\hline Malate-Aspartate Shuttle & 10 & 1 & 0.0879 & 0.0849 & 1.0 & 0.609 \\
\hline Warburg Effect & 58 & 2 & 0.51 & 0.0878 & 1.0 & 0.609 \\
\hline Glycine and Serine Metabolism & 59 & 2 & 0.519 & 0.0905 & 1.0 & 0.609 \\
\hline $\begin{array}{l}\text { Valine, Leucine and Isoleucine } \\
\text { Degradation }\end{array}$ & 60 & 2 & 0.527 & 0.0932 & 1.0 & 0.609 \\
\hline Tryptophan Metabolism & 60 & 2 & 0.527 & 0.0932 & 1.0 & 0.609 \\
\hline $\begin{array}{l}\text { Phosphatidylethanolamine } \\
\text { Biosynthesis }\end{array}$ & 12 & 1 & 0.105 & 0.101 & 1.0 & 0.619 \\
\hline Phosphatidylcholine Biosynthesis & 14 & 1 & 0.123 & 0.117 & 1.0 & 0.674 \\
\hline $\begin{array}{l}\text { Beta Oxidation of Very Long Chain } \\
\text { Fatty Acids }\end{array}$ & 17 & 1 & 0.149 & 0.14 & 1.0 & 0.679 \\
\hline Ethanol Degradation & 19 & 1 & 0.167 & 0.156 & 1.0 & 0.679 \\
\hline Lactose Synthesis & 20 & 1 & 0.176 & 0.163 & 1.0 & 0.679 \\
\hline Betaine Metabolism & 21 & 1 & 0.185 & 0.171 & 1.0 & 0.679 \\
\hline Carnitine Synthesis & 22 & 1 & 0.193 & 0.178 & 1.0 & 0.679 \\
\hline $\begin{array}{l}\text { Transfer of Acetyl Groups into } \\
\text { Mitochondria }\end{array}$ & 22 & 1 & 0.193 & 0.178 & 1.0 & 0.679 \\
\hline
\end{tabular}




\begin{tabular}{|c|c|c|c|c|c|c|}
\hline Glycerolipid Metabolism & 25 & 1 & 0.22 & 0.2 & 1.0 & 0.679 \\
\hline Glycolysis & 25 & 1 & 0.22 & 0.2 & 1.0 & 0.679 \\
\hline Cysteine Metabolism & 26 & 1 & 0.229 & 0.207 & 1.0 & 0.679 \\
\hline $\begin{array}{l}\text { Oxidation of Branched Chain Fatty } \\
\text { Acids }\end{array}$ & 26 & 1 & 0.229 & 0.207 & 1.0 & 0.679 \\
\hline $\begin{array}{l}\text { Mitochondrial Beta-Oxidation of } \\
\text { Short Chain Saturated Fatty Acids }\end{array}$ & 27 & 1 & 0.237 & 0.215 & 1.0 & 0.679 \\
\hline $\begin{array}{l}\text { Phenylalanine and Tyrosine } \\
\text { Metabolism }\end{array}$ & 28 & 1 & 0.246 & 0.222 & 1.0 & 0.679 \\
\hline Selenoamino Acid Metabolism & 28 & 1 & 0.246 & 0.222 & 1.0 & 0.679 \\
\hline $\begin{array}{l}\text { Mitochondrial Beta-Oxidation of } \\
\text { Long Chain Saturated Fatty Acids }\end{array}$ & 28 & 1 & 0.246 & 0.222 & 1.0 & 0.679 \\
\hline Phospholipid Biosynthesis & 29 & 1 & 0.255 & 0.229 & 1.0 & 0.679 \\
\hline Folate Metabolism & 29 & 1 & 0.255 & 0.229 & 1.0 & 0.679 \\
\hline Lysine Degradation & 30 & 1 & 0.264 & 0.236 & 1.0 & 0.679 \\
\hline Ammonia Recycling & 32 & 1 & 0.281 & 0.249 & 1.0 & 0.698 \\
\hline Beta-Alanine Metabolism & 34 & 1 & 0.299 & 0.263 & 1.0 & 0.714 \\
\hline Gluconeogenesis & 35 & 1 & 0.308 & 0.27 & 1.0 & 0.714 \\
\hline $\begin{array}{l}\text { Nicotinate and Nicotinamide } \\
\text { Metabolism }\end{array}$ & 37 & 1 & 0.325 & 0.283 & 1.0 & 0.73 \\
\hline Sphingolipid Metabolism & 40 & 1 & 0.352 & 0.302 & 1.0 & 0.732 \\
\hline Propanoate Metabolism & 42 & 1 & 0.369 & 0.315 & 1.0 & 0.732 \\
\hline Methionine Metabolism & 43 & 1 & 0.378 & 0.321 & 1.0 & 0.732 \\
\hline Histidine Metabolism & 43 & 1 & 0.378 & 0.321 & 1.0 & 0.732 \\
\hline Fatty acid Metabolism & 43 & 1 & 0.378 & 0.321 & 1.0 & 0.732 \\
\hline Pyruvate Metabolism & 48 & 1 & 0.422 & 0.352 & 1.0 & 0.784 \\
\hline Arginine and Proline Metabolism & 53 & 1 & 0.466 & 0.381 & 1.0 & 0.831 \\
\hline Arachidonic Acid Metabolism & 69 & 1 & 0.606 & 0.468 & 1.0 & 0.996 \\
\hline Tyrosine Metabolism & 72 & 1 & 0.633 & 0.483 & 1.0 & 1.0 \\
\hline Purine Metabolism & 74 & 1 & 0.65 & 0.492 & 1.0 & 1.0 \\
\hline
\end{tabular}


Table S2: Summary table for the metabolite set enrichment analysis (MSEA) are ranked by Holm p-value. The Holm $p$-value is the p-value adjusted by the Holm-Bonferroni test, which is a tool used for largescale data analysis to counteract the issue of multiple comparisons. At left side, the yellow to red colors summarizes metabolic pathways that were lowest to strongest affected respectively in the ReA compared to RA patients.

\begin{tabular}{|c|c|c|c|c|c|c|}
\hline Metabolite Set & Total & Hits & Expect & $P$ value & Holm P & FDR \\
\hline Urea Cycle & 29 & 2 & 0.198 & 0.0149 & 1.0 & 0.699 \\
\hline Amino Sugar Metabolism & 33 & 2 & 0.226 & 0.0191 & 1.0 & 0.699 \\
\hline Aspartate Metabolism & 35 & 2 & 0.239 & 0.0214 & 1.0 & 0.699 \\
\hline Pyruvate Metabolism & 48 & 2 & 0.328 & 0.0389 & 1.0 & 0.792 \\
\hline Glutamate Metabolism & 49 & 2 & 0.335 & 0.0404 & 1.0 & 0.792 \\
\hline Warburg Effect & 58 & 2 & 0.396 & 0.0551 & 1.0 & 0.841 \\
\hline Phenylacetate Metabolism & 9 & 1 & 0.0615 & 0.0601 & 1.0 & 0.841 \\
\hline Ketone Body Metabolism & 13 & 1 & 0.0889 & 0.0858 & 1.0 & 0.912 \\
\hline Glucose-Alanine Cycle & 13 & 1 & 0.0889 & 0.0858 & 1.0 & 0.912 \\
\hline $\begin{array}{l}\text { Beta Oxidation of Very Long } \\
\text { Chain Fatty Acids }\end{array}$ & 17 & 1 & 0.116 & 0.111 & 1.0 & 0.912 \\
\hline Alanine Metabolism & 17 & 1 & 0.116 & 0.111 & 1.0 & 0.912 \\
\hline Ethanol Degradation & 19 & 1 & 0.13 & 0.123 & 1.0 & 0.912 \\
\hline Glutathione Metabolism & 21 & 1 & 0.144 & 0.135 & 1.0 & 0.912 \\
\hline Carnitine Synthesis & 22 & 1 & 0.15 & 0.141 & 1.0 & 0.912 \\
\hline Glycerolipid Metabolism & 25 & 1 & 0.171 & 0.159 & 1.0 & 0.912 \\
\hline $\begin{array}{l}\text { Oxidation of Branched Chain } \\
\text { Fatty Acids }\end{array}$ & 26 & 1 & 0.178 & 0.165 & 1.0 & 0.912 \\
\hline $\begin{array}{l}\text { Mitochondrial Beta-Oxidation of } \\
\text { Short Chain Saturated Fatty Acids }\end{array}$ & 27 & 1 & 0.185 & 0.171 & 1.0 & 0.912 \\
\hline Selenoamino Acid Metabolism & 28 & 1 & 0.191 & 0.177 & 1.0 & 0.912 \\
\hline $\begin{array}{l}\text { Mitochondrial Beta-Oxidation of } \\
\text { Long Chain Saturated Fatty Acids }\end{array}$ & 28 & 1 & 0.191 & 0.177 & 1.0 & 0.912 \\
\hline Ammonia Recycling & 32 & 1 & 0.219 & 0.2 & 1.0 & 0.952 \\
\hline Gluconeogenesis & 35 & 1 & 0.239 & 0.217 & 1.0 & 0.952 \\
\hline Fatty Acid Biosynthesis & 35 & 1 & 0.239 & 0.217 & 1.0 & 0.952 \\
\hline $\begin{array}{l}\text { Nicotinate and Nicotinamide } \\
\text { Metabolism }\end{array}$ & 37 & 1 & 0.253 & 0.228 & 1.0 & 0.952 \\
\hline Galactose Metabolism & 38 & 1 & 0.26 & 0.233 & 1.0 & 0.952 \\
\hline Fatty acid Metabolism & 43 & 1 & 0.294 & 0.26 & 1.0 & 1.0 \\
\hline
\end{tabular}




\begin{tabular}{|l|l|l|l|l|l|l|l|l|l|}
\hline \multicolumn{1}{|c|}{ Metabolite Set } & \multicolumn{1}{c|}{ Total } & Hits & \multicolumn{1}{c|}{ Expect } & P value & Holm P & FDR \\
\hline & Glycine and Serine Metabolism & 59 & 1 & 0.403 & 0.341 & 1.0 & 1.0 \\
\hline & Pyrimidine Metabolism & 59 & 1 & 0.403 & 0.341 & 1.0 & 1.0 \\
\hline & Tryptophan Metabolism & 60 & 1 & 0.41 & 0.346 & 1.0 & 1.0 \\
\hline & Purine Metabolism & 74 & 1 & 0.506 & 0.409 & 1.0 & 1.0 \\
\hline
\end{tabular}


Table S3: Pearson correlation table $(n=38)$ of metabolites present in SF and serum

\begin{tabular}{|c|c|c|c|c|}
\hline \multirow[t]{2}{*}{ S.No. } & \multirow[t]{2}{*}{ Metabolite } & \multicolumn{2}{|c|}{ Metabolite Ratios (mean \pm SD ) } & \multirow{2}{*}{$\begin{array}{c}\text { Pearson } \\
\text { Cofficient } \\
\text { (r) }\end{array}$} \\
\hline & & SF & Serum & \\
\hline 1 & 2-Methylglutarate & $1.11 \pm 0.55$ & $1.78 \pm 1.71$ & 0.1899 \\
\hline 2 & 3-Hydroxybutyrate & $25.70 \pm 27.90$ & $22.89 \pm 25.35$ & 0.785 \\
\hline 3 & 3-Methyl-2-oxovalerate & $1.74 \pm 0.81$ & $2.26 \pm 2.08$ & 0.2057 \\
\hline 4 & Acetate & $19.93 \pm 7.67$ & $26.96 \pm 12.34$ & 0.7052 \\
\hline 5 & Acetone & $10.13 \pm 11.76$ & $9.319 \pm 7.59$ & 0.7306 \\
\hline 6 & Alanine & $84.68 \pm 31.82$ & $133.36 \pm 52.95$ & 0.4638 \\
\hline 7 & Betaine & $17.41 \pm 9.64$ & $18.90 \pm 12.83$ & 0.8673 \\
\hline 8 & Carnitine & $9.91 \pm 3.93$ & $10.97 \pm 5.84$ & 0.3098 \\
\hline 9 & Choline & $4.65 \pm 2.54$ & $7.00 \pm 3.44$ & 0.5095 \\
\hline 10 & Citrate & $21.31 \pm 6.96$ & $15.19 \pm 8.10$ & -0.1723 \\
\hline 11 & Creatine & $6.22 \pm 4.40$ & $6.91 \pm 4.52$ & 0.7626 \\
\hline 12 & Creatinine & $13.41 \pm 5.78$ & $15.16 \pm 7.13$ & 0.56 \\
\hline 13 & Dimethyl sulfone & $1.73 \pm 0.83$ & $2.15 \pm 1.25$ & 0.356 \\
\hline 14 & Ethanol & $22.81 \pm 19.96$ & $228.08 \pm 678.74$ & 0.3578 \\
\hline 15 & Fucose & $6.10 \pm 2.33$ & $10.41 \pm 7.26$ & -0.0219 \\
\hline 16 & Glucose & $1191.61 \pm 415.96$ & $1423.54 \pm 599.33$ & 0.4332 \\
\hline 17 & Glutamate & $23.37 \pm 18.78$ & $40.80 \pm 18.31$ & 0.187 \\
\hline 18 & Glutamine & $106.5 \pm 43.26$ & $134.01 \pm 57.55$ & 0.4022 \\
\hline 19 & Glycerol & $81.55 \pm 136.31$ & $104.24 \pm 243.03$ & 0.6147 \\
\hline 20 & Glycine & $73.17 \pm 33.70$ & $109.80 \pm 58.96$ & 0.6972 \\
\hline 21 & Histidine & $18.16 \pm 6.88$ & $24.99 \pm 10.88$ & 0.4763 \\
\hline 22 & Isobutyrate & $1.36 \pm 0.59$ & $1.73 \pm 0.90$ & 0.5196 \\
\hline 23 & Isoleucine & $20.23 \pm 5.80$ & $22.93 \pm 9.06$ & 0.2024 \\
\hline 24 & Isopropanol & $6.97 \pm 16.41$ & $41.75 \pm 126.20$ & 0.7286 \\
\hline 25 & Lactate & $672.84 \pm 279.52$ & $1013.64 \pm 424.70$ & 0.1495 \\
\hline 26 & Leucine & $38.89 \pm 12.96$ & $47.42 \pm 18.00$ & 0.1483 \\
\hline 27 & Lysine & $34.16 \pm 13.00$ & $26.48 \pm 15.63$ & 0.3891 \\
\hline 28 & Mannose & $20.57 \pm 7.08$ & $26.91 \pm 13.91$ & 0.3551 \\
\hline
\end{tabular}




\begin{tabular}{|l|l|l|l|l|}
\hline 29 & Methanol & $22.27 \pm 12.44$ & $38.21 \pm 33.99$ & 0.3786 \\
\hline 30 & Methionine & $8.53 \pm 3.04$ & $7.97 \pm 3.76$ & 0.2546 \\
\hline 31 & Phenylalanine & $15.89 \pm 6.12$ & $20.86 \pm 11.71$ & 0.44 \\
\hline 32 & Proline & $57.90 \pm 22.51$ & $67.52 \pm 32.69$ & 0.4815 \\
\hline 33 & Pyruvate & $20.54 \pm 7.79$ & $10.89 \pm 9.11$ & 0.04577 \\
\hline 34 & Serine & $24.41 \pm 10.77$ & $49.70 \pm 18.43$ & 0.2875 \\
\hline 35 & Threonine & $32.12 \pm 13.00$ & $42.51 \pm 17.81$ & 0.3117 \\
\hline 36 & Tyrosine & $15.89 \pm 4.50$ & $16.30 \pm 6.53$ & 0.3822 \\
\hline 37 & Valine & $58.04 \pm 17.57$ & $63.05 \pm 23.48$ & 0.3822 \\
\hline 38 & Myoinositol & $6.56 \pm 2.30$ & $9.93 \pm 3.91$ & 0.5572 \\
\hline
\end{tabular}


Table S4: Significnat metabolites of ReA compare to non-ReA groupswith p-value, false discovery rate (FDR).

\begin{tabular}{|c|c|c|c|c|c|c|}
\hline \multirow{2}{*}{ S.No } & \multirow{2}{*}{ Metabolite } & \multirow{2}{*}{ ppm } & \multicolumn{2}{|c|}{ ReA vs OA } & \multicolumn{2}{|c|}{ ReA vs RA } \\
\hline & & & $p$ value & FDR & $p$ value & FDR \\
\hline 1 & $\mathrm{LDL}$ & 0.85 & 0.005 & 0.0055066 & -- & -- \\
\hline 2 & Isoleucine $\$$ & 0.91 & 0.000 & 6.07E-06 & -- & -- \\
\hline 3 & $3-\mathrm{HB}$ & 1.18 & 0.010 & 0.01117 & -- & -- \\
\hline 4 & VLDL & 1.25 & 0.049 & 0.048766 & -- & -- \\
\hline 5 & Alanine $\$$ & 1.46 & 0.000 & $2.35 \mathrm{E}-06$ & 0.00 & $6.16 \mathrm{E}-06$ \\
\hline 6 & Acetate & 1.89 & 0.000 & 6.07E-06 & 0.00 & $5.43 E-05$ \\
\hline 7 & NAG\$ & 2.01 & 0.000 & $6.82 \mathrm{E}-07$ & -- & -- \\
\hline 8 & Glutamate $\$$ & 2.05 & 0.000 & $5.18 \mathrm{E}-07$ & -- & -- \\
\hline 9 & Acetone & 2.21 & -- & -- & -- & -- \\
\hline 10 & Glutamine & 2.43 & -- & -- & 0.00 & 0.002008 \\
\hline 11 & Choline & 3.18 & 1.08 & -- & -- & -- \\
\hline 12 & Carnitine $\$$ & 3.20 & 0.003 & 0.0042582 & 0.00 & $1.75 \mathrm{E}-06$ \\
\hline 13 & Glucose $\$$ & 3.38 & 0.000 & $3.14 \mathrm{E}-05$ & & \\
\hline 14 & Glycerol\$ & 3.55 & 0.002 & 0.0026877 & 0.01 & 0.014396 \\
\hline 15 & Lactate & 4.09 & -- & -- & 0.00 & 0.001288 \\
\hline
\end{tabular}

\$Represent the diagnostic panel of biomarkers as inferred from their significantly changed levels in ReA compared to nonReA groups 
Figure S1: Representative $800 \mathrm{MHz}{ }^{1} \mathrm{H}$ NMR spectra $\delta(0.75-4.65)(\mathrm{A})$ and $\delta(5.2-8.6)$ (B) of synovial fluid obtained from the OA, RA and ReA groups. The region of $\delta(5.2-8.5)$ is magnified 8 times compared with the corresponding region of $\delta(0.5-4.7)$ for the purpose of clarity. Abbreviations and notions used are: $3-$ HB: 3-Hydroxybutyrate; NAG: N-acetylated glycoprotein; LDL: Low Density Lipoprotein; VLDL: Very Low Density Lipoprotein; PUFA: Polyunsaturated Fatty Acid $-\mathrm{C} \underline{\mathrm{H}}=\mathrm{C} \underline{\mathrm{H}}-; \mathrm{L} 1:-\mathrm{C}_{2}-\mathrm{CH}_{2}-\mathrm{C}=\mathrm{O}-; \mathrm{L} 2:-\underline{\mathrm{H}}_{2}-\mathrm{C}=\mathrm{O}-$; L3: $=\mathrm{CH}-\mathrm{C}_{2}-\mathrm{CH}=\mathrm{C}-$.

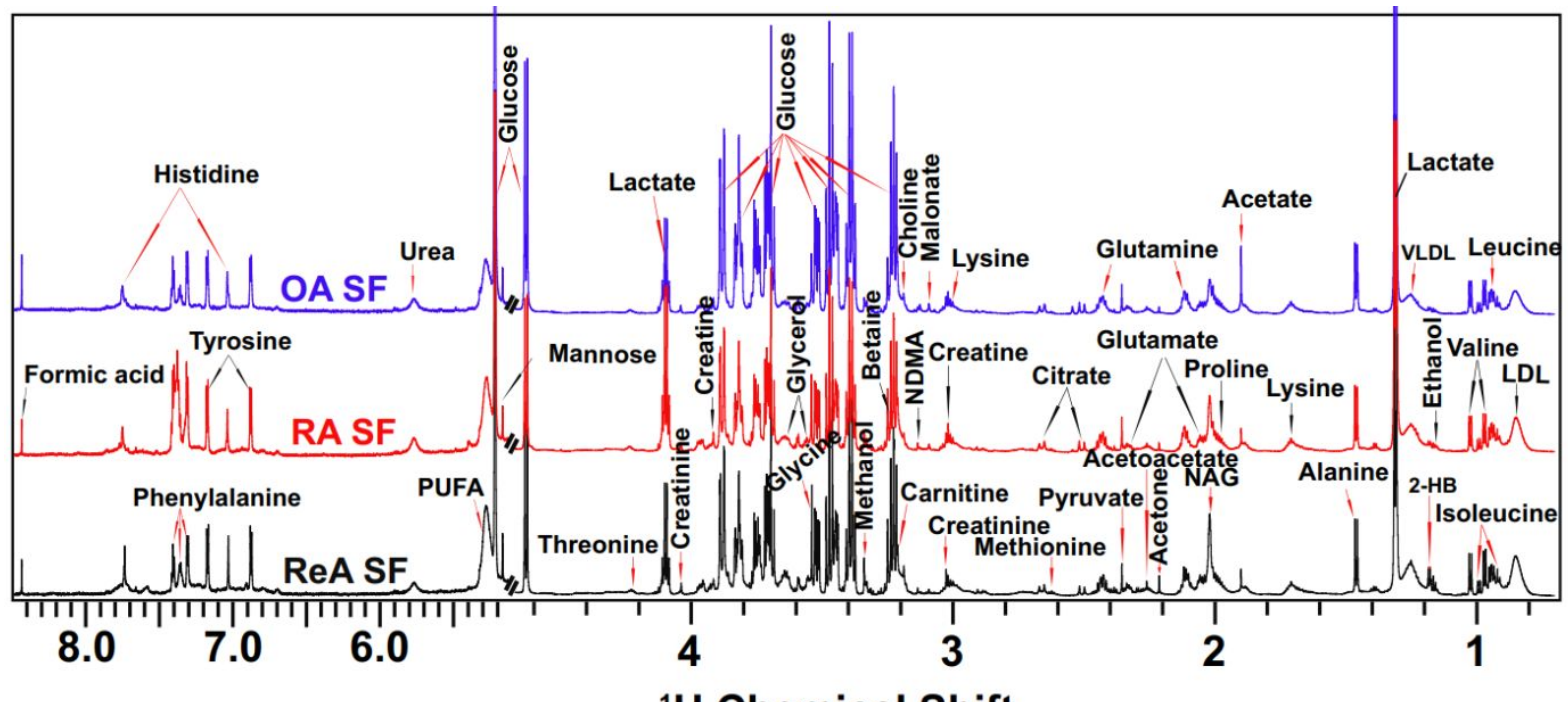

${ }^{1} \mathrm{H}$ Chemical Shift 
Figure S2: The statistical validation plots (permutation number $=100$ ) for OPLS-DA model based discriminatory analysis (A) between ReA and RA $\left(R^{2} X(\right.$ cum $)=0.739, \quad R^{2} Y($ cum $)=0.671$, and $\left.\mathrm{Q}^{2}(\mathrm{cum})=0.514\right)$. (B) between ReA and $\mathrm{OA}\left(\mathrm{R}^{2} \mathrm{X}(\mathrm{cum})=0.723, \mathrm{R}^{2} \mathrm{Y}(\mathrm{cum})=0.754\right.$, and $\left.\mathrm{Q}^{2}(\mathrm{cum})=0.0 .604\right)$
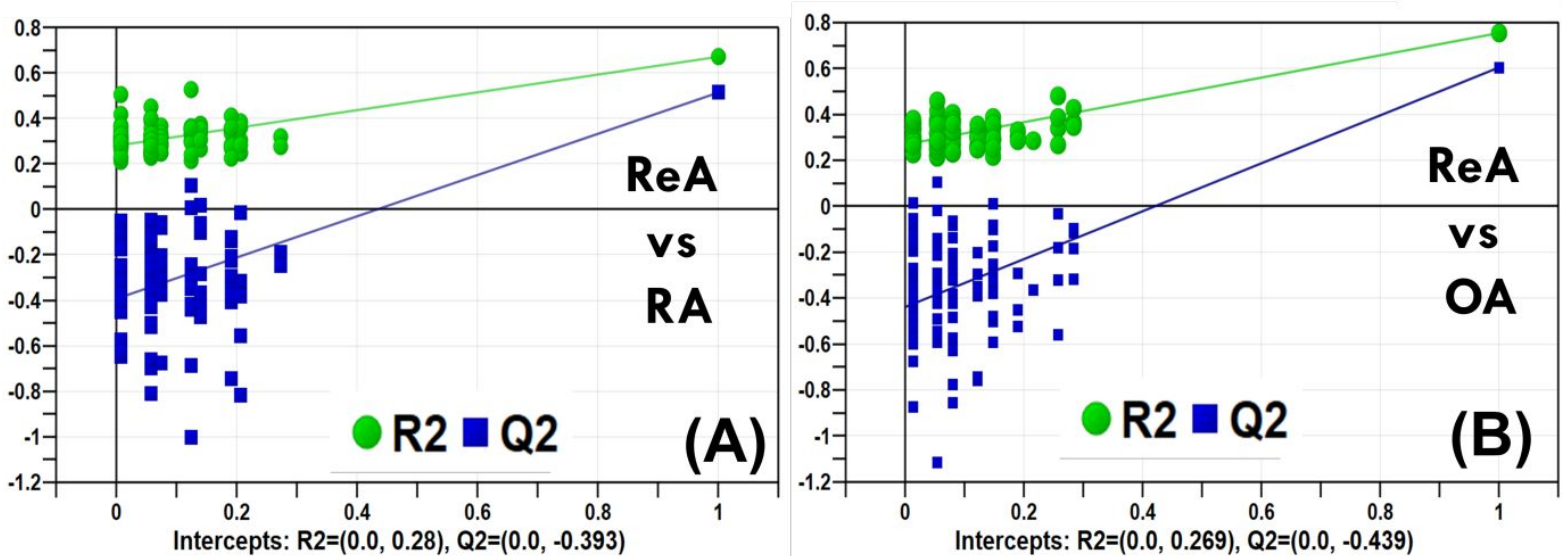

Figure S3: Significantly altered metabolic pathways in ReA patient compare to OA and RA. All matched pathways are shown according to $p$ values from the pathway enrichment analysis ( $y$ axis) and pathway impact values from the pathway topology analysis ( $x$ axis). Colour gradient and circle size indicate the significance of the pathway ranked by $p$ value (yellow: higher $p$ values and red: lower $p$ values) and pathway impact values (the larger the circle the higher the impact score). Only the significantly affected pathways with low $p$ value and high pathway impact score are showed.

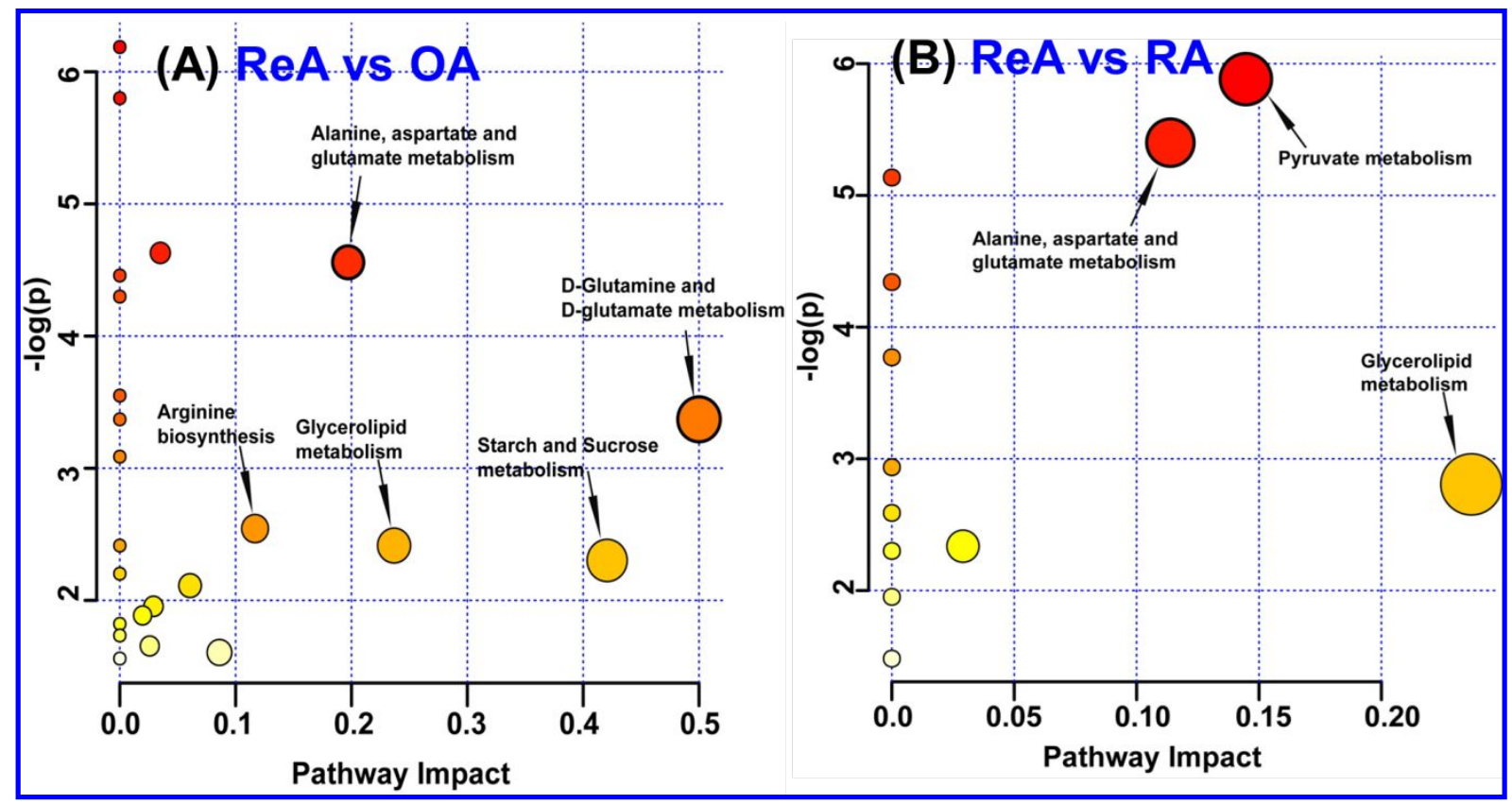


Figure S4: Metabolic pathway analyses related to the metabolites that significantly differ in ReA compared to nor-ReA group, utilizing the MetaboAnalyst functional interpretation tools. Panel A, graphic summary of metabolite set enrichment analysis for ReA vs OA groups. Panel B, graphic summary of metabolite set enrichment analysis for ReA vs RA groups. The horizontal bars summarize the main metabolite sets identified in this analysis; the bars are coloured based on their p-values and the length is based on the -fold enrichment.

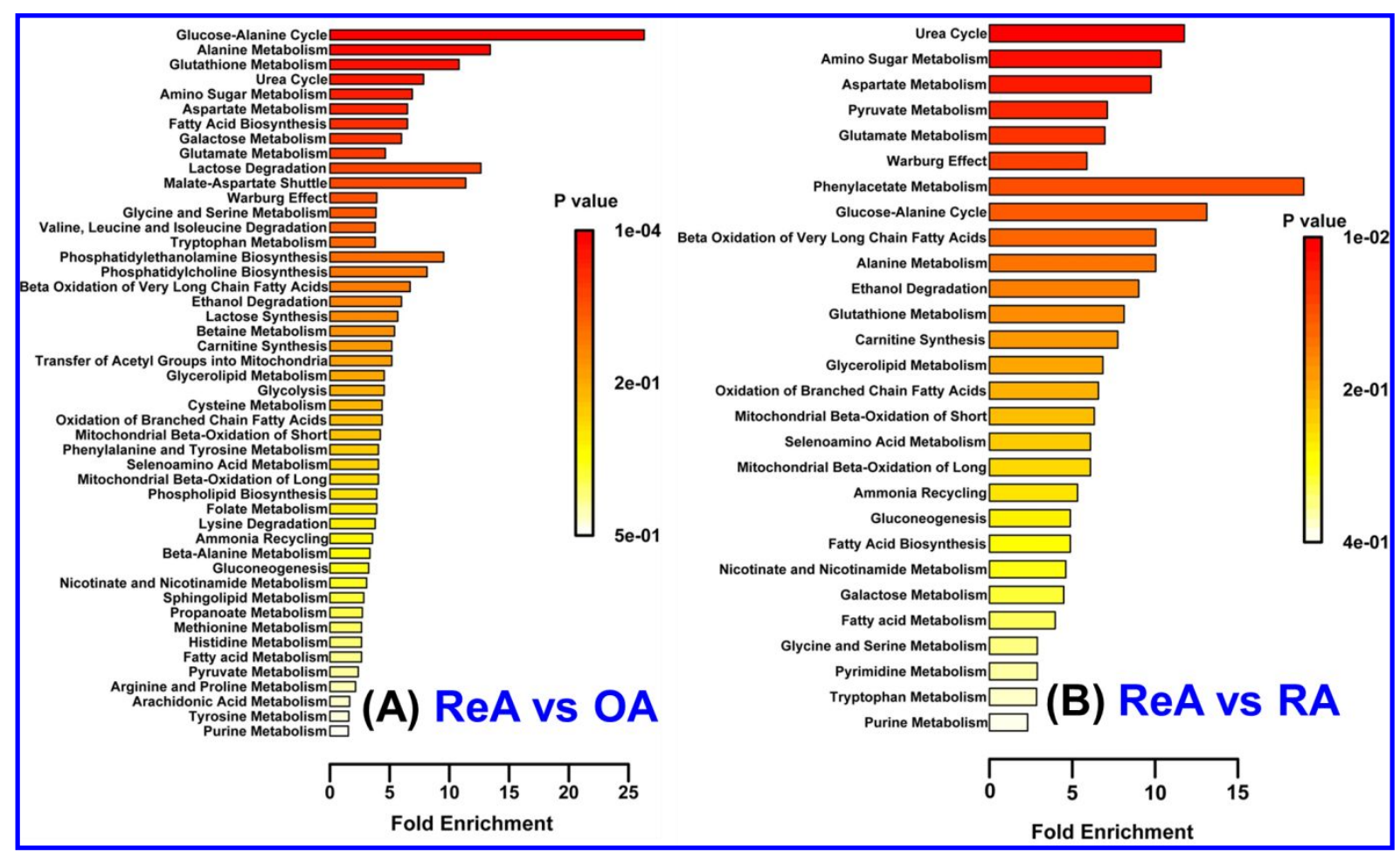


Figure S5: Potential biomarkers of (A) ReA vs OA and (B) ReA vs RA predicted by classical univariate ROC curve analysis. The left-side plot of the individual metabolite figure shows a 95\% confidence interval (shadowed) ROC (receiver operating characteristics) curve and the solid red dot shows the optimum cutoff with the corresponding sensitivity and specificity values. The right-side box-and-whisker plot shows the distribution of abundance values of individual metabolite in ReA compare to non-ReA group with the optimal cutoff as a horizontal dotted red line.

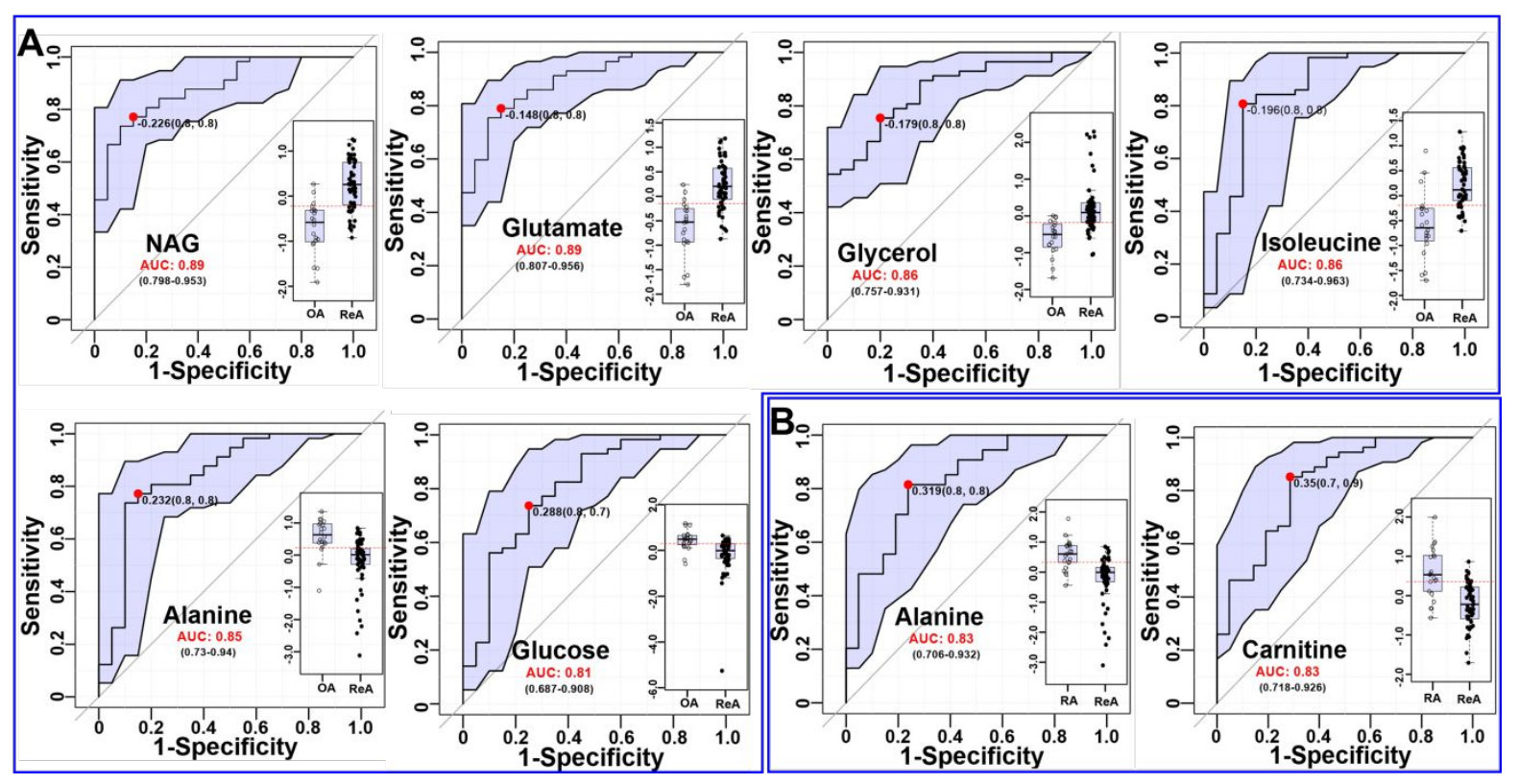


Figure S6: The percentage selected frequency of metabolites based on ROC curves, with the variable importance in projection (VIP) plot from ReA vs RA (A) and ReA vs RA (C) indicating the most discriminating metabolite in descending order of importance; prediction of ReA patients from (B) RA and (D) OA groups using Monte-Carlo cross validation (MCCV) analysis. The class membership of the leftout sample was predicted using an a priori cut-off value of 0.5 (dashed line).

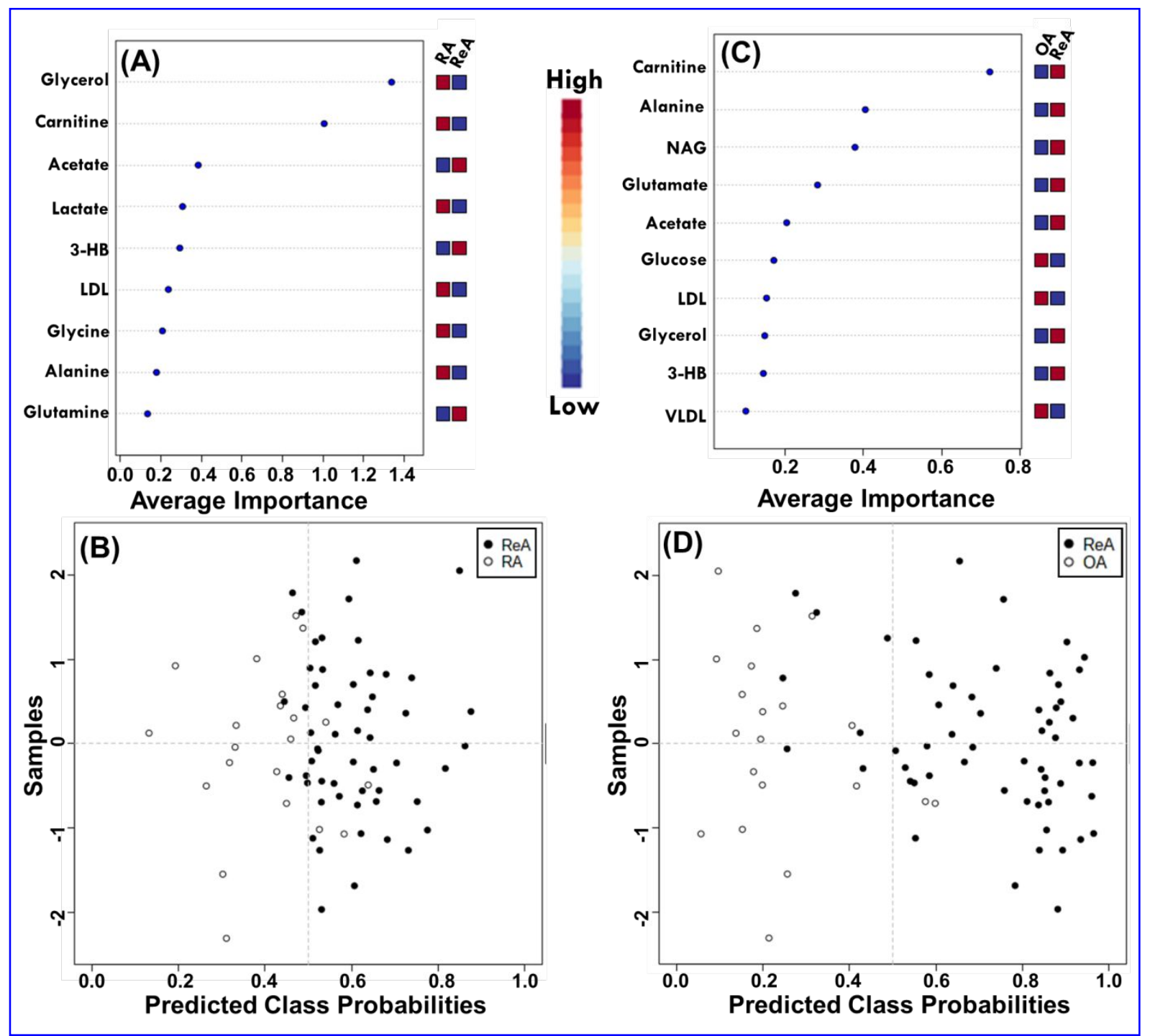

